\title{
Composición Corporal y Somatotipo Referencial de Sujetos Físicamente Activos
}

\author{
Body Composition and Referential Somatotype of Physically Active Subjects \\ "Fernando Javier Rodríguez Rodríguez; **Atilio Aldo Almagià Flores; **Tuillang Yuing Farias; \\ **** Octavio Binvignat Gutierrez \& **Pablo Lizana Arce
}

RODRÍGUEZ, R. F. J.; AlMAgì̀, F. A. A.; YUING, F. T.; BINVIGNAT, G. O. \& LIZANA, A. P. Composición corporal y somatotipo referencial de sujetos físicamente activos. Int. J. Morphol., 28(4):1159-1165, 2010.

RESUMEN: En nuestro país y en el mundo se han realizado una serie de investigaciones en el área de la morfoestructura humana, pero pocos trabajos que describan el perfil antropométrico de personas jóvenes saludables. Se evaluaron a 100 hombres y 79 mujeres de entre 20 y 29 años de edad, sin factores de riesgo a la salud. Se evaluó de acuerdo a un protocolo de ISAK con variables que permite estimar la composición corporal y el somatotipo de los individuos. De esta manera se presentan las tablas de resultados como una forma de usarlas de referencia. Nuestro grupo de referencia evaluado (CHIREF) con sus resultados de composición corporal, somatotipo e índices corporales, aporta una nueva fuente de información de la V Región y actualizada para Chile, la cual favorecerá las comparaciones para distintos grupos de edades, condiciones de salud, deportes y etnias, considerando la necesidad de aumentar el grupo etario y la cantidad de variables antropométricas para ampliar el universo de comparación y mejorar la referencia comparativa.

PALABRAS CLAVE: Antropometría; Composición corporal; Somatotipo.

\section{INTRODUCCIÓN}

En Chile se han realizado una serie de investigaciones en el área de la antropometría, utilizando distintos métodos de evaluación y aplicados a variados tipos de poblaciones, como deportistas de alto rendimiento, amateur, jóvenes deportistas, sedentarios, obesos, diabéticos y de distintas etnias, realizando estudios descriptivos para cada población (Almagià et al., 1986; Almagià et al., 1996a; Almagià et al., 1996b; Almagià et al., 1997; Almagià et al., 2008; Almagià et al., 2009; Silva et al., 2003; Silva et al., 2005). Pero no existen trabajos en nuestro país que describan la morfoestructura humana de individuos jóvenes, sanos y activos que no sean deportistas, pero que se encuentran dentro de este grupo etario, y que permitan estimar una referencia comparativa para salud y actividad física, objetivo que trataremos de dilucidar en el desarrollo de este trabajo.

\section{MATERIAL Y MÉTODO}

Los sujetos evaluados fueron principalmente alumnos universitarios y voluntarios, quienes accedieron al estudio como una muestra no aleatoria e intencionada. Estos voluntarios debían tener una buena salud, y realizar actividad física recreacional, (una o dos veces por semana), sin factores de riesgo cardiovascular y con un IMC normal, lo cual se determinó previo a la evaluación. Se excluyeron además las personas con obesidad y extranjeros residentes.

La muestra está formada por 100 hombres y 79 mujeres entre 20 y 29 años de edad. El criterio de exclusión para menores de 20 años y más de 29 años está referido a que el punto central de comparación de nuestro estudio son los deportistas, quienes se encuentran mayoritariamente entre esos rangos de edad.

\footnotetext{
Laboratorio de Motricidad Humana, Escuela de Educación Física, Facultad de Filosofia y Educación Laboratorio de Antropología Física y Anatomía Humana, Instituto de Biología, Facultad de Ciencias, , Pontificia Universidad Católica de Valparaíso, Chile.

** Laboratorio de Antropología Física y Anatomía Humana, Instituto de Biología, Facultad de Ciencias, Pontificia Universidad Católica de Valparaíso, Chile.

*** Centro Anatómico, Campus Talca, Universidad Autónoma. Chile; Profesor colaborador Laboratorio de Antropología Física y Anatomía Humana, Instituto de Biología, Facultad de Ciencias, Pontificia Universidad Católica de Valparaíso, Chile.
} 
El grupo de estudio pertenece a la V Región cordillera y V Región Costa de Chile y la elección de los sujetos fue de manera no probabilística.

Para determinar el Somatotipo (Carter, 1980) y la Composición Corporal Método (Kerr, 1988) se midieron las siguientes variables 25 variables antropométricas, 3 variables básicas que son el peso, la estatura y la estatura sentado, 6 pliegues cutáneos: tricipital, subescapular, supraespinal, abdominal, de la parte frontal del muslo y pierna medial. Además, de 7 perímetros musculares: del brazo relajado corregido por el pliegue cutáneo tricipital, brazo contraído, del antebrazo, de la cabeza,de la cintura, perímetro del tórax, perímetro del muslo máximo, perímetro muslo medio y perímetro de la pierna y 7 diámetros óseos: biacromial, anteroposterior, biiliocrestideo, biepicondilar del húmero, biepicondilar del fémur, antero-posterior de la caja torácica y transversal del tórax.
Se utilizó para la estimación del Somatotipo, el método de Heath- Carter (Carter, 1984, 2002) y para determinar la composición corporal, se utilizó el método de Kerr que consiste en el fraccionamiento corporal en cinco componentes masa muscular, masa ósea, masa grasa, masa piel y masa residual. Se asegura la validez de la evaluación realizándose por un evaluador, quien ha obtenido el Nivel II ISAK (International Society for the Avancement in Kineanthropometry), de esta forma se valida la metodología utilizada y se disminuyen los errores. Para la aplicación del método antropométrico se utilizó el Kit Gaucho Pro "Mercosur", fabricado en Argentina bajo licencia de Rosscraft Canadá. Se utilizó un asistente que ayudó al evaluador a anotar los datos, dicho asistente conocía las técnicas de medición lo que permitió un trabajo fluido, ya que aseguraba la secuencia correcta de los sitios de medición. También para minimizar los errores de la medición, se tra-

Tabla I. Resultados de las variables y resultados estadísticos del grupo de evaluado (CHIREF) de hombres n= 100 .

\begin{tabular}{|c|c|c|c|c|c|c|c|c|c|c|c|c|c|}
\hline \multirow{2}{*}{\multicolumn{2}{|c|}{ CHI REF Hombres }} & \multicolumn{5}{|c|}{ Estadísticas } & \multicolumn{7}{|c|}{ Percentiles } \\
\hline & & Prom & $\mathrm{D} \mathrm{S}$ & Mediana & Mín & Máx & $5 \%$ & $15 \%$ & $25 \%$ & $50 \%$ & $75 \%$ & $85 \%$ & $95 \%$ \\
\hline \multirow{4}{*}{$\sum_{\infty}^{\infty}$} & Edad (años) & 21,1 & 1,8 & 20,0 & 20,0 & 29,0 & 20,0 & 20,0 & 20,0 & 20,0 & 22,0 & 22,2 & 25,1 \\
\hline & Peso (kg) & 69,2 & 8,1 & 68,7 & 47,2 & 93,2 & 58,4 & 61,5 & 63,9 & 68,7 & 73,4 & 77,6 & 84,2 \\
\hline & Talla (cm) & 173,3 & 7,3 & 173,0 & 160,5 & 197,0 & 164,5 & 166,0 & 167,6 & 173,0 & 177,3 & 179,1 & 186,1 \\
\hline & Talla sentado $(\mathrm{cm})$ & 90,9 & 4,1 & 91,0 & 82,0 & 103,0 & 84,2 & 86,3 & 88,0 & 91,0 & 93,9 & 95,0 & 98,1 \\
\hline \multirow{6}{*}{ 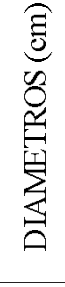 } & Biacromial & 39,9 & 2,3 & 40,1 & 31,3 & 44,3 & 36,3 & 38,3 & 38,5 & 40,1 & 41,6 & 42,0 & 43,2 \\
\hline & Tórax transverso & 28,9 & 1,6 & 29,0 & 25,5 & 34,3 & 26,3 & 27,5 & 27,9 & 29,0 & 29,9 & 30,4 & 31,8 \\
\hline & Tórax anteroposterior & 19,4 & 3,5 & 18,9 & 14,6 & 40,5 & 16,2 & 17,5 & 18,0 & 18,9 & 19,9 & 20,5 & 22,2 \\
\hline & Bi-iliocrestídeo & 27,7 & 1,5 & 27,9 & 24,9 & 30,9 & 25,1 & 25,7 & 26,5 & 27,9 & 28,6 & 29,3 & 30,1 \\
\hline & Humeral (biepicondilar) & 6,9 & 0,4 & 6,9 & 5,9 & 7,8 & 6,3 & 6,6 & 6,6 & 6,9 & 7,1 & 7,3 & 7,6 \\
\hline & Femoral (biepicondilar) & 9,8 & 0,5 & 9,8 & 8,8 & 11,1 & 9,0 & 9,4 & 9,5 & 9,8 & 10,1 & 10,3 & 10,5 \\
\hline \multirow{10}{*}{ 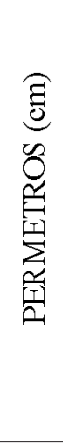 } & Cabeza & 55,7 & 1,3 & 55,6 & 53,2 & 59,5 & 53,7 & 54,2 & 55,0 & 55,6 & 56,5 & 57,0 & 57,7 \\
\hline & Brazo relajado & 29,6 & 2,5 & 29,3 & 23,7 & 36,5 & 26,0 & 27,1 & 27,8 & 29,3 & 31,1 & 32,0 & 34,1 \\
\hline & Brazo flexionado tensión & 31,4 & 2,8 & 31,5 & 25,0 & 38,9 & 27,6 & 28,6 & 29,2 & 31,5 & 33,3 & 34,0 & 37,0 \\
\hline & Antebrazo & 26,6 & 1,6 & 26,5 & 23,2 & 31,5 & 24,3 & 25,0 & 25,5 & 26,5 & 27,4 & 28,2 & 29,2 \\
\hline & Tórax mesoesternal & 95,2 & 6,3 & 94,5 & 68,3 & 114,4 & 86,5 & 89,3 & 91,5 & 94,5 & 99,4 & 100,8 & 104,1 \\
\hline & Cintura (mínima) & 79,0 & 6,7 & 78,3 & 49,5 & 99,5 & 71,1 & 73,3 & 75,1 & 78,3 & 83,3 & 85,6 & 88,0 \\
\hline & Caderas (máxima) & 94,1 & 4,9 & 93,0 & 86,1 & 109,4 & 87,6 & 89,5 & 90,5 & 93,0 & 97,0 & 99,0 & 102,5 \\
\hline & Muslo (superior) & 57,8 & 6,2 & 56,5 & 49,3 & 85,2 & 51,7 & 53,5 & 54,5 & 56,5 & 59,0 & 61,0 & 65,4 \\
\hline & Muslo (medial) & 51,7 & 3,5 & 51,6 & 40,8 & 59,8 & 46,6 & 48,2 & 49,6 & 51,6 & 54,3 & 55,1 & 57,7 \\
\hline & Pierna (máxima) & 36,8 & 2,0 & 36,7 & 30,7 & 41,9 & 34,1 & 34,7 & 35,3 & 36,7 & 38,4 & 38,9 & 40,2 \\
\hline \multirow{6}{*}{ 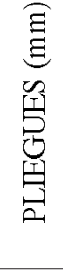 } & Tríceps & 8,9 & 4,9 & 8,0 & 2,0 & 27,0 & 3,0 & 4,0 & 6,0 & 8,0 & 11,0 & 14,0 & 19,5 \\
\hline & Subescapular & 9,2 & 4,0 & 8,5 & 3,0 & 27,0 & 5,0 & 6,0 & 7,0 & 8,5 & 10,9 & 12,0 & 15,0 \\
\hline & Supraespinal & 5,5 & 4,2 & 5,0 & 1,0 & 23,0 & 1,0 & 2,0 & 3,0 & 5,0 & 7,0 & 8,3 & 13,8 \\
\hline & Abdominal & 16,6 & 10,0 & 13,8 & 3,5 & 50,0 & 5,0 & 8,0 & 9,3 & 13,8 & 21,8 & 28,0 & 35,8 \\
\hline & Muslo (medial) & 6,5 & 3,8 & 6,0 & 1,0 & 17,0 & 1,0 & 3,0 & 4,0 & 6,0 & 9,0 & 11,0 & 13,1 \\
\hline & Pierna & 4,7 & 2,5 & 5,0 & 1,0 & 13,0 & 1,0 & 2,0 & 3,0 & 5,0 & 6,0 & 7,0 & 8,0 \\
\hline
\end{tabular}


bajó en una instrucción previa con los asistentes y se efectuaron otras evaluaciones a modo de práctica para coordinar de mejor manera el trabajo en equipo, y de esta forma, la recolección de datos fuera sin errores y expedita, dando énfasis sobre todo en la lectura y anotación de datos. La ficha de recolección de los datos fue diseñada con la intención de mejorar la rapidez de la medición, con un orden que va desde superior a inferior de la posición anatómica. Los datos fueron registrados por anotadores a través de planillas en papel, datos que posteriormente eran traspasados a planillas de registro hechas en Microsoft Office Excel 2003.

\section{RESULTADOS}

Una vez traspasados los datos se procedió a calcular los resultados de composición corporal, Somatotipo e IMC. Estas formulas están incluidas en un software "Antropogym" que facilitaba el cálculo de los resultados. Las estadísticas calculadas fueron, promedio, desviación estándar, mediana, mínimo, máximo y percentiles 5\%, 15\%, 25\%, 50\%, 75\%, 85\% y $95 \%$, siendo estas, en antropometría, las medidas más utilizadas para propósitos de ubicación o clasificación de los individuos cuando atienden a características tales como peso, estatura, etc. Las tablas de los resultados son presentadas en valores estadísticos que son; promedios, desvíos estándar, mediana, valor mínimo, valor máximo, percentiles 5, 15, 25, $50,75,85$ y 95 .

Comparando los resultados de composición corporal entre hombres y mujeres, apreciamos resultados esperables de los principales componentes, definidos por factores hormonales. Un porcentaje promedio de masa grasa de $21,6+$ 4,1 para hombres y 29,6+4,2 para mujeres, una diferencia de $30 \%$ más de masa grasa en mujeres que en hombres. No pre- ocupa la diferencia entre ambos géneros, dada la conocida funcionalidad del tejido adiposo en mujeres, pero si el elevado porcentaje graso que compone la morfoestructura femenina de este grupo especifico.

Se presenta igualmente el gráfico de comparación de los componentes de la composición corporal entre ambos géneros.

Con respecto al Somatotitpo de Heath-Carter (Carter, 1984), el cual establece diferencia en la forma y que reflejan la adiposidad, robustez músculo esquelética y linealidad relativas a la talla, se obtuvo una clasificación tanto para hombres como para mujeres de endomesomórficos, lo que quiere decir que predomina el componente mesomórfico, secundariamente el componente endomórfico y terciariamente el componente ectomórfico.

Esta somatocarta muestra una amplia distribución espacial del grupo mujeres, pero que se concentran hacia la izquierda (endomorfismo) y cercanas también al punto medio de somatotipo balanceado. Lo alejado de los puntos hacia el componente ectomórfico, denota características morfológicas robustas.

La figura 3, muestra que la mayoría de los individuos hombres se encuentran en el tercio superior de la somatorcarta, alejados del endomorfismo y con alguna tendencia al ectomorfismo, es decir clasificados con predominio del mesomorfismo, o sea, robustez de la forma corporal por medio de la masa muscular. El IMC expresados en $\mathrm{kg} / \mathrm{m} 2$ son $23,0+2,3$ para hombres y 22,5 + 3,2 para mujeres, valores que según el Centers for Disease Control and Prevention (CDC) y World Health Organization (WHO) de EEUU, se caracterizan como saludables o dentro del rango de peso normal (entre 18,5 y $24,9 \mathrm{~kg} / \mathrm{m}^{2}$ ).

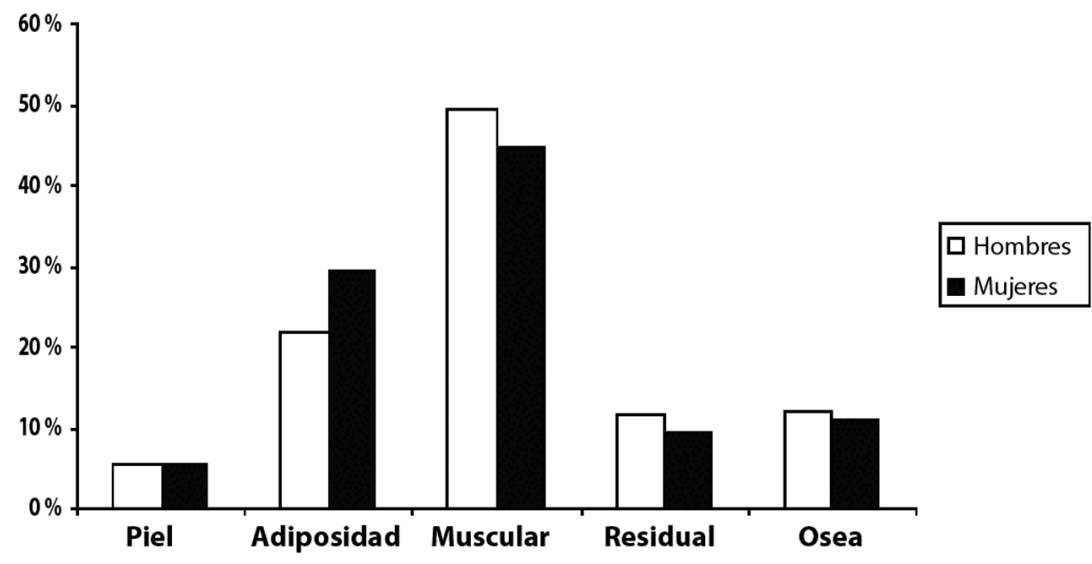

Fig. 1. Comparación de los cinco componentes de la composición corporal establecidos según el método de D. Kerr, 1988 entre hombres y mujeres de la muestra. 
RODRÍGUEZ, R. F. J.; ALMAGIÀ, F. A. A.; YUING, F. T.; BINVIGNAT, G. O. \& LIZANA, A. P. Composición corporal y somatotipo referencial de sujetos físicamente activos.

Int. J. Morphol., 28(4):1159-1165, 2010.

Tabla II. Resultados de las variables y resultados estadísticos del grupo de evaluado (CHIREF) de mujeres, $\mathrm{n}=79$.

\begin{tabular}{|c|c|c|c|c|c|c|c|c|c|c|c|c|c|}
\hline \multirow{2}{*}{\multicolumn{2}{|c|}{ CHIREF Mujeres }} & \multirow[b]{2}{*}{ Prom } & \multicolumn{4}{|c|}{ Estadísticas } & \multicolumn{6}{|c|}{ Percentiles } & \multirow[b]{2}{*}{$95 \%$} \\
\hline & & & $\mathrm{DS}$ & Mediana & Min & Max & $5 \%$ & $15 \%$ & $25 \%$ & $50 \%$ & $75 \%$ & $85 \%$ & \\
\hline \multirow{4}{*}{ 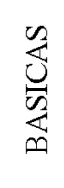 } & Edad (años) & 21,2 & 1,9 & 21,0 & 20,0 & 29,0 & 20,0 & 20,0 & 20,0 & 21,0 & 22,0 & 23,0 & 25,0 \\
\hline & Peso $(\mathrm{kg})$ & 58,3 & 8,7 & 56,8 & 41,3 & 84,0 & 46,1 & 50,4 & 52,9 & 56,8 & 62,5 & 66,3 & 74,3 \\
\hline & Talla (cm) & 161,0 & 5,2 & 161,0 & 151,0 & 174,0 & 153,0 & 155,6 & 157,0 & 161,0 & 165,0 & 166,0 & 169,2 \\
\hline & Talla sentado $(\mathrm{cm})$ & 85,6 & 3,5 & 86,0 & 77,8 & 93,5 & 79,9 & 82,0 & 83,0 & 86,0 & 88,0 & 89,0 & 90,6 \\
\hline \multirow{6}{*}{ 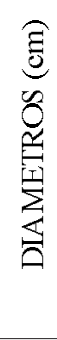 } & Biac romial & 35,9 & 1,6 & 35,9 & 31,5 & 39,7 & 33,6 & 34,5 & 35,0 & 35,9 & 36,8 & 37,6 & 38,5 \\
\hline & Tórax transverso & 26,1 & 2,4 & 25,7 & 18,3 & 35,4 & 23,5 & 24,5 & 25,0 & 25,7 & 27,0 & 27,5 & 28,7 \\
\hline & Tórax anteroposterior & 16,6 & 1,4 & 16,6 & 13,5 & 20,5 & 14,6 & 15,2 & 15,6 & 16,6 & 17,5 & 18,0 & 18,7 \\
\hline & Bi-iliocrestídeo & 27,2 & 3,1 & 27,3 & 5,9 & 36,0 & 24,3 & 25,4 & 26,4 & 27,3 & 28,8 & 29,3 & 30,0 \\
\hline & $\begin{array}{l}\text { Humeral } \\
\text { (biepicondilar) }\end{array}$ & 5,9 & 0,3 & 5,9 & 5,5 & 6,7 & 5,5 & 5,6 & 5,7 & 5,9 & 6,2 & 6,3 & 6,5 \\
\hline & (biepicondilar) & 8,8 & 0,4 & 8,8 & 8,0 & 10,0 & 8,2 & 8,5 & 8,6 & 8,8 & 9,0 & 9,1 & 9,4 \\
\hline \multirow{10}{*}{ 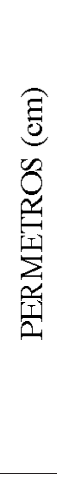 } & Cabeza & 53,9 & 1,1 & 53,9 & 50,7 & 56,0 & 52,1 & 52,7 & 53,2 & 53,9 & 54,9 & 55,0 & 55,8 \\
\hline & Brazo relajado & 27,0 & 2,4 & 26,6 & 21,5 & 33,7 & 23,6 & 24,6 & 25,3 & 26,6 & 28,4 & 29,2 & 31,0 \\
\hline & $\begin{array}{l}\text { Brazo flexionado } \\
\text { tensión }\end{array}$ & 27,7 & 2,3 & 27,3 & 22,7 & 35,6 & 24,5 & 25,7 & 26,3 & 27,3 & 29,3 & 29,9 & 32,0 \\
\hline & Antebrazo & 23,4 & 1,5 & 23,3 & 20,3 & 28,5 & 21,2 & 21,9 & 22,2 & 23,3 & 24,6 & 25,0 & 25,8 \\
\hline & Tórax mesoesternal & 87,4 & 5,3 & 87,0 & 72,5 & 100,4 & 79,8 & 82,2 & 84,4 & 87,0 & 90,3 & 92,5 & 96,6 \\
\hline & Cintura (mínima) & 71,4 & 6,4 & 70,8 & 51,1 & 87,3 & 63,4 & 66,0 & 67,0 & 70,8 & 75,1 & 77,5 & 84,2 \\
\hline & Caderas (máxima) & 95,1 & 6,7 & 94,3 & 81,8 & 113,0 & 85,0 & 88,5 & 90,7 & 94,3 & 99,7 & 101,8 & 106,9 \\
\hline & Muslo (superior) & 57,2 & 5,9 & 56,5 & 47,2 & 85,7 & 49,4 & 51,7 & 53,5 & 56,5 & 60,6 & 61,7 & 67,5 \\
\hline & Muslo (medial) & 49,4 & 4,1 & 49,6 & 40,5 & 59,0 & 42,4 & 45,5 & 46,5 & 49,6 & 52,0 & 53,2 & 56,5 \\
\hline & Pierna (máxima) & 35,1 & 3,0 & 35,5 & 28,9 & 46,4 & 30,3 & 31,9 & 33,2 & 35,5 & 37,0 & 37,6 & 38,9 \\
\hline \multirow{6}{*}{ 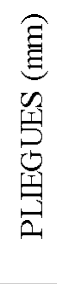 } & Tríceps & 16,3 & 5,3 & 16,0 & 6,0 & 30,0 & 8,0 & 10,0 & 12,5 & 16,0 & 20,0 & 22,0 & 25,1 \\
\hline & Subescapular & 11,1 & 5,1 & 10,0 & 3,0 & 28,0 & 5,0 & 6,0 & 8,0 & 10,0 & 14,0 & 16,3 & 20,1 \\
\hline & Supraespinal & 11,1 & 5,5 & 10,0 & 2,0 & 32,0 & 4,0 & 5,4 & 7,0 & 10,0 & 14,0 & 16,3 & 21,1 \\
\hline & Abdominal & 23,2 & 8,5 & 22,0 & 5,5 & 39,0 & 8,8 & 15,0 & 18,0 & 22,0 & 28,5 & 32,6 & 38,1 \\
\hline & Muslo (medial) & 14,1 & 6,1 & 13,0 & 1,0 & 28,0 & 5,0 & 9,0 & 11,0 & 13,0 & 17,5 & 21,3 & 25,1 \\
\hline & Pierna & 10,1 & 4,6 & 10,0 & 1,0 & 20,0 & 3,0 & 5,0 & 6,0 & 10,0 & 13,5 & 15,0 & 18,0 \\
\hline
\end{tabular}

Tabla III. Valores promedios y su desviación estándar del somatotipo para hombres y mujeres expresados en valores numéricos enteros según Carter \& Heath, 1990.

\begin{tabular}{lll}
\hline \multirow{2}{*}{ Somatotipo } & \multicolumn{2}{l}{ CHIREF } \\
\cline { 2 - 3 } Endo & Varones & Damas \\
Meso & $2,7 \pm 3,6$ & $4,1 \pm 1,3$ \\
Ecto & $5,1 \pm 1$ & $4,2 \pm 1,1$ \\
$\mathrm{X}$ & $2,5+1,2$ & $2,1+1,1$ \\
$\mathrm{Y}$ & $0,1 \pm 0,1$ & $-2,0 \pm 2,2$ \\
\hline
\end{tabular}




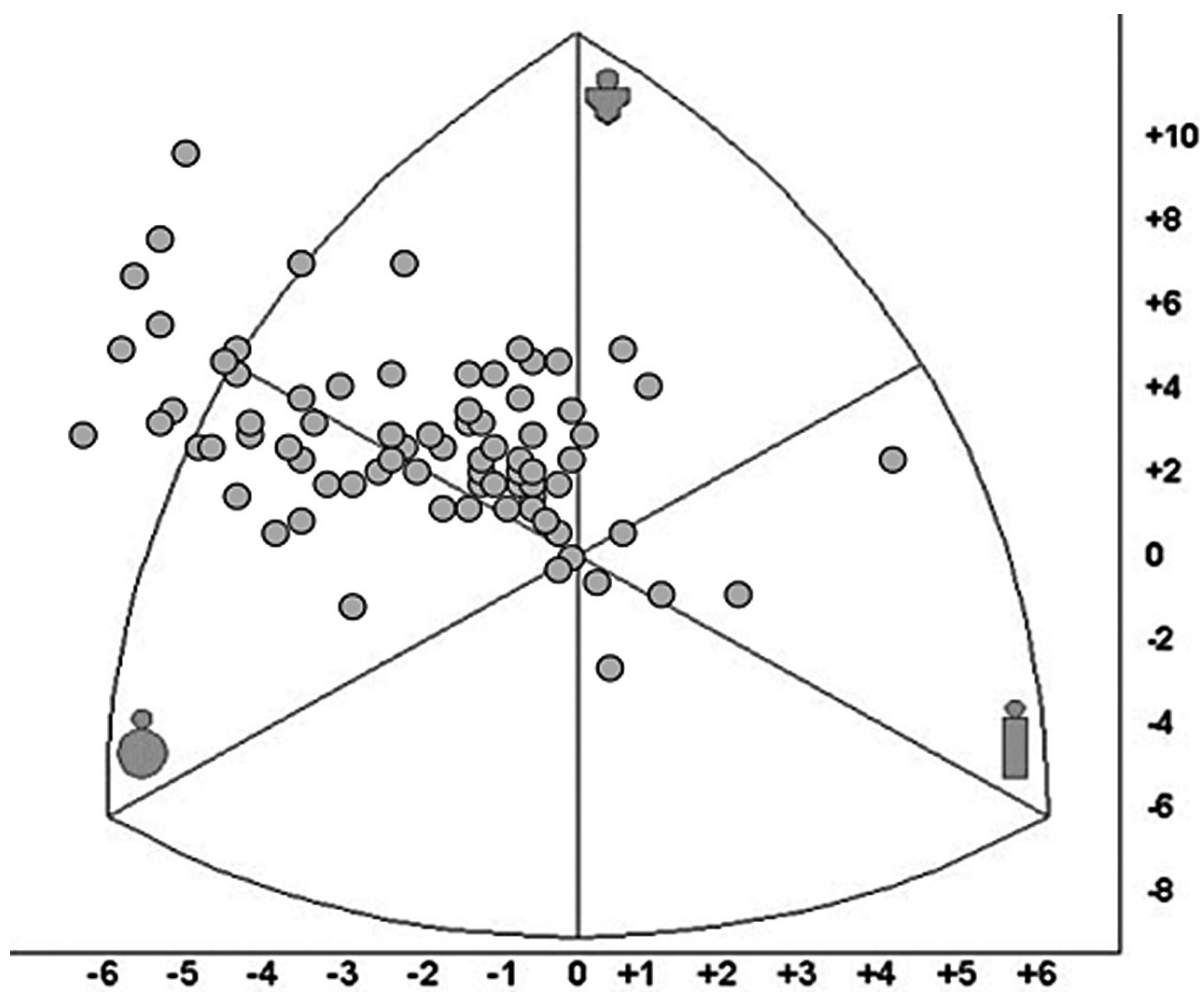

Fig. 2. Distribución espacial de la somatocarta de Somatotipo de mujeres CHIREF, con $n=79$

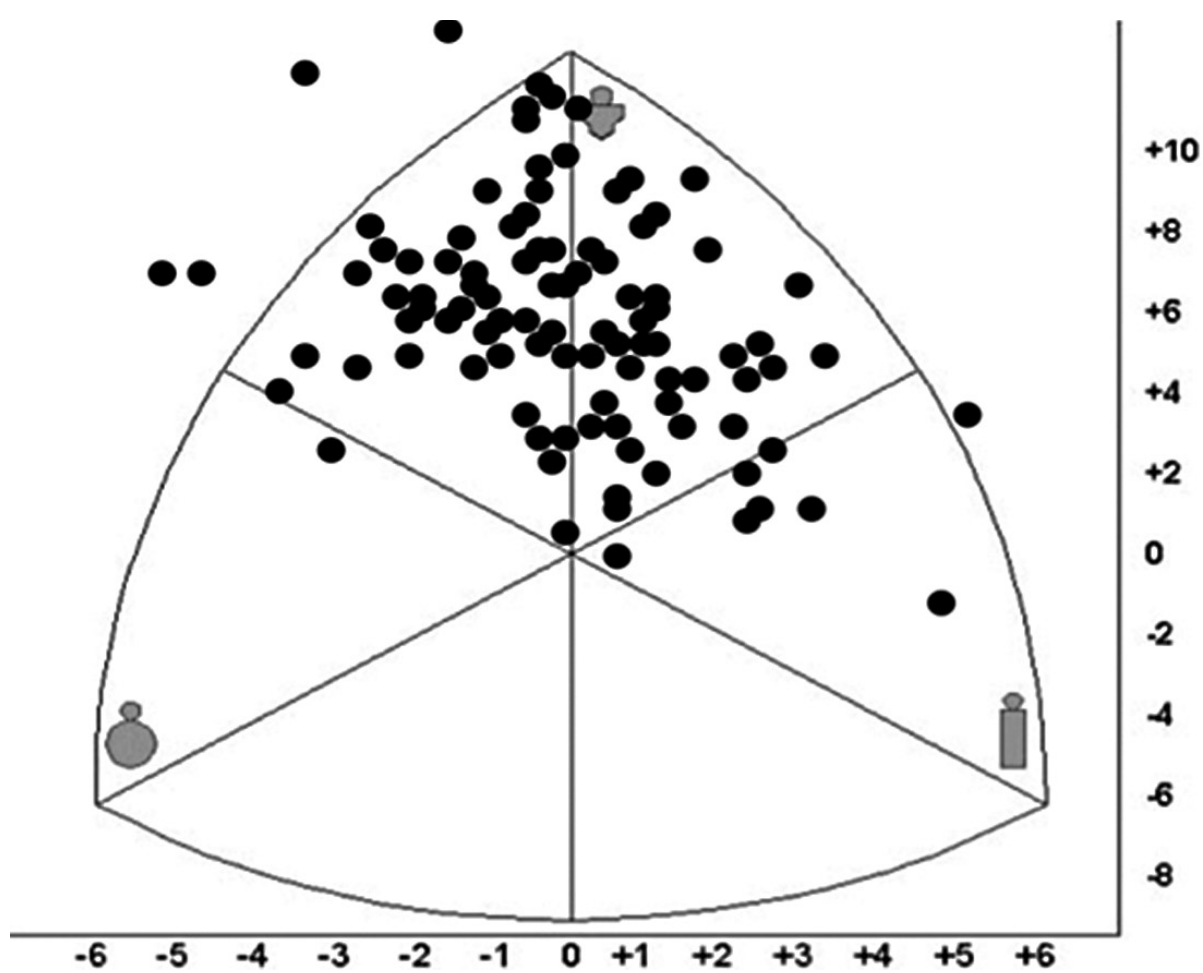

Fig. 3. Distribución espacial de la somatocarta de Somatotipo de hombres CHIREF, con un $n=$ 100. 


\section{DISCUSIÓN}

El establecimiento de un modelo antropométrico comparativo, toma un gran valor al momento de poder contrastar evaluaciones hechas a individuos que desarrollan distintas actividades físicas o condicionadas a factores que puedan modificar la morfoestructura humana. El hecho de establecer este "modelo comparativo" favorece además el conocimiento de las condiciones antropométricas en las que se encuentra este grupo de sujetos jóvenes de este rango etáreo, sirviendo de referencia no sólo para individuos con similares características, sino que también para comparar individuos de otras edades, niveles deportivos y condiciones nutricionales.

El aporte de los resultados como fuente de información actualizada y que a pesar de ser de la $\mathrm{V}$ región de Chile, sirve de gran ayuda para el desarrollo de nuestra disciplina de trabajo, agregándose como un referente para otros autores.

Se presentan las variables antropométricas como valores per se para una mejor compararación, como se realiza con el método Phantom (Ross \& Wilson, 1974; De Garay et al., 1974; Norton \& Olds, 1996).

Finalmente con respecto a la antropometría simple, el IMC, se presenta en todos los sujetos, dentro de los valores "normales y saludables" (WHO, 2000) y que la muestra intencionada no está contaminada con valores de IMC extremos, como desnutrición u obesidad.

La referencia del IMC, composición corporal y Somatotipo de este grupo característico sirve de gran ayuda para futuras investigaciones como valor comparable en sujetos jóvenes, normales y sanos. Las tablas que se presentan, nos entregan una valiosa información que beneficia el conocimiento de los profesionales ligados a la salud y el deporte, siendo esta, una muestra comparativa para futuras investigaciones.
La estimación de los componentes de la morfoestructura seccionados en cinco partes, favorece la especificidad de la muestra y permite establecer comparaciones más adecuadas y precisas, comparado con otros métodos antropométricos de dos o tres componentes.

Según la clasificación laboratorio Biosystem Argentina, los hombres se encuentran con un porcentaje de grasa considerado como excelente, en cambio las mujeres entran en la categoría de tejido adiposo aceptable, considerando que la tabla de referencia está diseñada para el sexo masculino.

Concluimos que el grupo CHIREF con sus resultados de composición corporal, Somatotipo e índices corporales, es una nueva fuente de información de la $\mathrm{V}$ región y actualizada para Chile, la cual favorecerá las comparaciones para distintos grupos de edades, condiciones de salud, deportes y etnias, siendo una herramienta adecuada para su utilización. Se deja abierta la posibilidad de aumentar el número de sujetos y mejorar de esta manera el grupo comparativo.

Instamos a generar mayor cantidad de datos y variables de distintos grupos etáreos y étnicos para ampliar el universo de comparación y mejorar la referencia comparativa.

\section{AGRADECIMIENTOS}

Por el apoyo y financiamiento otorgados, a la Dirección de Investigación, Vice Rectoría de Investigación y Estudios Avanzados de la Pontificia Universidad Católica de Valparaíso, Chile.

RODRÍGUEZ, R. F. J.; ALMAGIì, F. A. A.; YUING, F. T.; BINVIGNAT, G. O. \& LIZANA, A. P. Body composition and referential somatotype of physically active subjects. Int. J. Morphol., 28(4):1159-1165, 2010.

SUMMARY: In our country and worldwide, extensive research has been carried out in the human morphostructure, however there is limited work that describes the anthropometric profile of young healthy individuals. One hundred men and seventy nine women were evaluated between 20 and 29 years of age without health risk factors. The evaluation was in accordance with ISAK protocol and variables in body composition estimate and somatotype. Reference tables of the results are also included. Reference group (CHIREF) with the results of body composition, somatotype and other corporal indexes contribute as a source of information from Chile, which will aid in comparison studies for different age groups, health conditions, sports and ethnicity, considering the need to increase the age group and the amount of anthropometric variables so as to expand the range of comparison and improve comparative referentes.

KEY WORDS: Anthropometry; Body composition; Somatotype. 


\section{REFERENCIAS BIBLIOGRAFICAS}

Almagià, F. A; Toro, D. T.; Binvignat, G. O.; Cabrera, E. \& Marinao, A. Aproximación al perfil morfoestructural y dismorfismo sexual de jóvenes de ambos sexos de edad de 6 a 23 años de edad caracterizados por el somatotipo. Rev. Chil. Anat., 14:189-97, 1996a.

Almagià, A.; Toro, T.; Gurovich A.; Cabrera, E.; Marinao, A. \& Binvignat, O. Determinación y análisis de la morfoestructura de un equipo de fútbol profesional. Rev. Chil. Anat., 14:87-95, 1996b.

Almagià, F. A. A.; Gurovich, M. A.; Ivanovic, M. D.; Toro, D. T. \& Binvignat, G. O. Estudio y análisis morfológico y etario del dimorfismo sexual a través de la composición corporal. Rev. Chil. Anat., 15:141-9, 1997.

Almagià, A.; Toro, T.; Costa, 1.; Silva, E. \& Díaz, C. Antropometría de damas y varones de 15 a 21 años de una muestra de estudiantes de Viña del Mar. An. Anat. Nor., 4:106-13, 1986.

Almagia, A.; Rodríguez, F.; Barraza, F.; Lizana, P.; Ivanovic, D. \& Birvignat, O. Perfil Antropométrico de Jugadores Profesionales de Voleibol Sudamericano. Int. J. Morphol., 27:53-7, 2009.

Almagia, A.; Rodríguez, F.; Barraza, F.; Lizana, P. \& Jorquera, C. Perfil Antropométrico de Jugadoras Chilenas de Fútbol Femenino. Int. J. Morphol., 26:817-21, 2008.

Carter, J. E. Somatotype of Olympic Athletes from 1948 to 1976. Med. Sports Sci., 18:80-109, 1980.

Carter J. E. L. The Heath-Carter Somatotype method. San Diego, San Diego State University Syllabus Service, 2002. De Garay, A. L.; Levine, L. \& Carter, J. E. L. Genetic and Anthropological Studies of Olympic Athletes. New York, Academic Press, 1974.

Carter, J. E. L. \& Heath, B. H. Somatotyping - Development and Applications. Cambridge: Cambridge University Press. 1990.

Kerr, D. A. An anthropometric method for the fractionation of skin, adipose, muscle, bone and residual tissue masses in males and females age 6 to 77 years. M.Sc. Thesis, Simon Fraser University, 1988.

Norton, K. \& Olds, T. Antropometrica. Marrickville, Sidney, Ed. Southwood Press, 1996.
Ross, W. D. \& Wilson, N. C. A stratagem for proportional growth assessment. Acta Paediatr. Belg., 28:169-82, 1974.

Silva, H.; Collipal, L. E.; Martínez, C. \& Bruneau, C. J. Evaluación de los Componentes del Somatotipo e Índice de Masa Corporal en Escolares del Sector Precordillerano de la IX Región, Chile. Int. J. Morphol., 23:195-9, 2005.

Silva, H.; Bruneau, J. C.; Reyno, H. P. \& Bucarey, S. Somatotipo e Índice de Masa Corporal en una muestra de adolescentes de ambos sexos de la ciudad de Temuco, Chile. Int. J. Morphol., 21:309-13, 2003.

WHO, 2000. World Health Report 2000. Geneva, World Health Organization, 2000.

Dirección para correspondencia:

Fernando Rodríguez Rodríguez

Avda. El Bosque 1290

Casilla 4059

Pontificia Universidad Católica de Valparaíso

CHILE

Email: fernando.rodriguez@ucv.cl

Recibido : 31-08-2010

Aceptado: 27-09-2010 
